\title{
Comparison of Resting Full Cycle Ratio with Fractional Flow Reserve in Indian Population
}

\author{
Shravan Kumar Ch $^{1}$ Sudhakar Kanumuri ${ }^{1} \quad$ Garre Indrani $^{1} \quad$ M Naveen Kumar ${ }^{1}$ \\ ${ }^{1}$ Department of Cardiology, Nizam's Institute of Medical Sciences, \\ Hyderabad, India \\ Address for correspondence Shravan Kumar Ch, DM, Department \\ of Cardiology, Nizam's Institute of Medical Sciences, Hyderabad, \\ Telangana 500 082, India (e-mail: shravankch@gmail.com).
}

Ind J Car Dis Wom:2020;5:95-99

\begin{abstract}
Background From the previous studies fractional flow reserve (FFR) was considered best for hemodynamic assessment of bordersline coronary lesions. In literature, previous studies compared instant wave-free ratio (IFR) with resting full cycle ratio (RFR) which concluded that both were equally effective diagnostically. Studies comparing RFR with FFR are still lacking in number.

So we have done a prospective study comparing RFR and FFR in predominately chronic stable angina patients, whose coronary angiogram suggestive of borderline coronary lesions.

Material and Methods In the department of cardiology in our institution, we have done a prospective study in chronic stable angina patients whose coronary angiogram was suggestive of borderline coronary lesions from March 2019 to June 2019. We recorded RFR value, basal FFR value, and after-adenosine FFR values in borderline coronary lesion patients.

Results A total of 33 patients were included in the study. The study population was predominantly male (93.9\%). Hypertension was noted in $81.8 \%$ and diabetes was noted in $51.5 \%$ patients. We compared RFR and FFR by regression analysis; it suggested that

Keywords

- chronic stable angina

- coronary angiogram

- FFR

- RFR both RFR and FFR were significantly correlated, with $p=0.000$. This is true even in subgroup analysis of $\operatorname{sex}(p=0.001)$.

Conclusion From our study, we conclude that RFR may be used instead of FFR for physiologic assessment of inducible ischemia in borderline coronary artery lesions as it is independent of gender also.
\end{abstract}

\section{Introduction}

From the previous studies fractional flow reserve (FFR) was considered as a gold standard for hemodynamic assessment of borderline coronary lesions. In literature, previous studies compared instant wave-free ratio (IFR) with resting full cycle ratio (RFR) which concluded that both are equally effective diagnostically. ${ }^{1,2}$ Studies comparing RFR with FFR are still lacking in number. RFR, which is independent of cardiac cycle length, may detect significant coronary stenosis which pertained to specific cardiac cycle segments.

We compared both RFR with FFR in this study. RFR may be considered better than FFR as it is independent of cardiac cycle and rhythm, and there is no need to use a coronary hyperemic agent.

\section{Aim}

The aim was to study the correlation between RFR and FFR in angiographically borderline coronary lesions in patients admitted to our hospital.

\section{Material and Methods}

In the department of cardiology in our institution, we have done prospective study in chronic stable angina patients whose coronary angiogram was suggestive of borderline coronary lesions from March 2019 to June 2019 to whom
C)2020 Women in Cardiology and Related Sciences
License terms

()(1) $\Theta \circledast$ 


\section{Abstract Image}

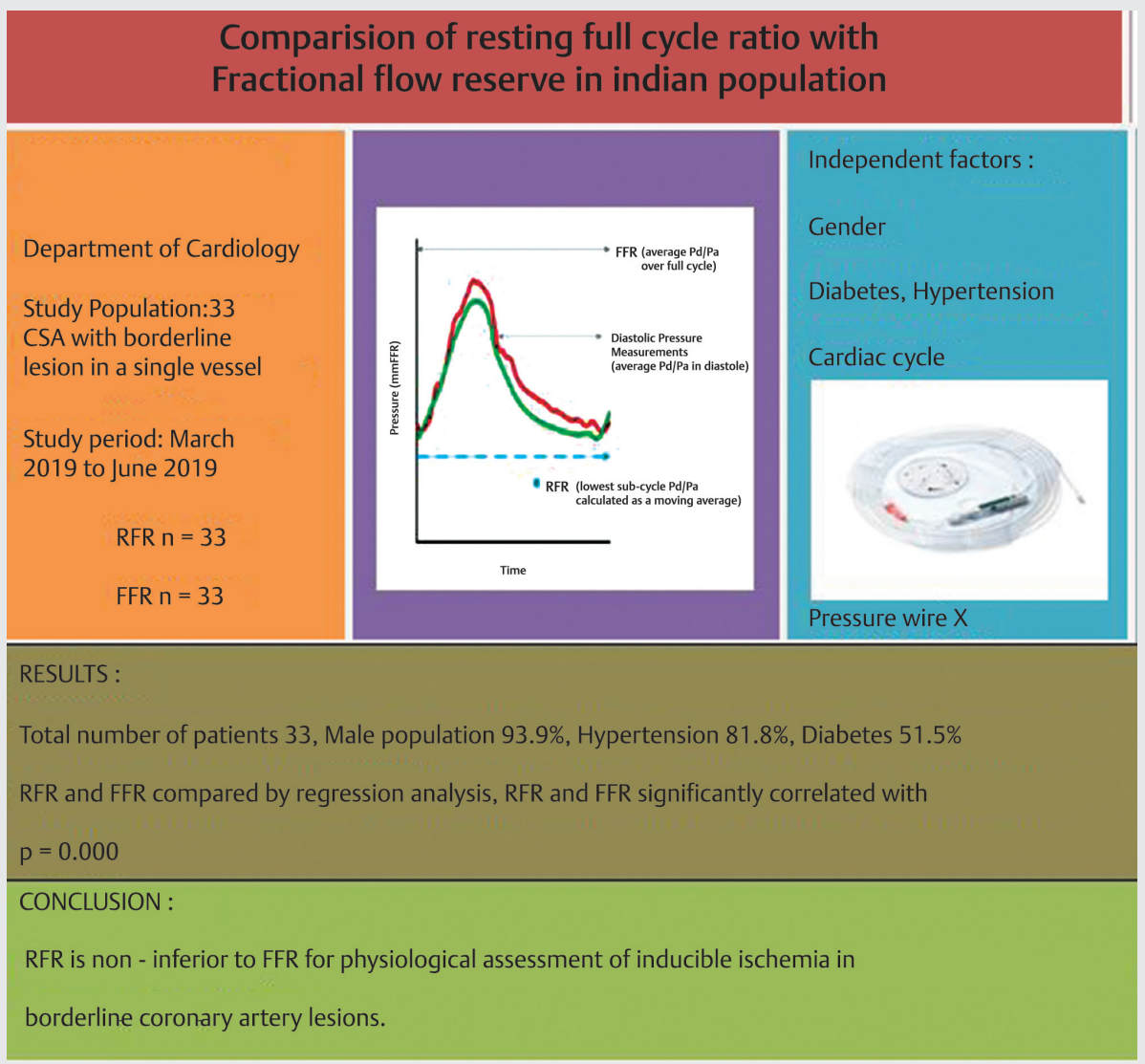

inducible ischemia assessment was to be done. Informed consentfrom the patients undergoing invasive coronary angiogram/ percutaneous coronary intervention, those who had borderline coronary lesions, were taken.

We used QUANTIEN Integrated RFR console (Abbott Global Health Care, United States) during the procedure to record FFR, RFR values. For the procedure FFR pressure wire $\mathrm{X}$ (St. Jude, United States) coronary wire was used. Procedure was done according to standard intervention guidelines. We equalized the pressure after pressure wire tip crossed the guiding catheter, and then it was advanced beyond the lesion of interest. Pressures were continuously recorded. We recorded RFR value, followed by FFR value after changing mode in console, intracoronary adenosine given and FFR value after adenosine was also recorded. These values have been analyzed for further intervention in the concerned patient.

All the data were incorporated in MS Excel, and baseline characteristics and laboratory parameters were analyzed, followed by regression analysis to compare FFR and RFR values. We divided the study population into subgroups, depending on gender and diabetes; the results were analyzed.

\section{Results}

A total of 33 patients were included in the study. The study population was predominantly male (93.9\%). Hypertension was noted in $81.8 \%$ and diabetes noted in $51.5 \%$. Borderline left anterior descending (LAD) coronary lesion was noted in 26 cases, right coronary artery (RCA) lesion in 5 cases, and left circumflex artery (LCX) lesion in 2 cases. Coronary lesions were classified as types $\mathrm{A}, \mathrm{B}$, and $\mathrm{C}$ according to the American College of Cardiology/American Heart Association guidelines. In the study population, type A lesions were noted in 25 patients and type B lesions noted in 9 patients. Baseline characteristics and laboratory parameters of the study population were included below in - Tables $\mathbf{1}$ and $\mathbf{2}$.

To compare the RFR and after-adenosine FFR values of the study population, we used regression analysis. Both RFR and

Table 1 Baseline characteristics of study population

\begin{tabular}{|l|l|}
\hline Variable & Value \\
\hline Age (years) & $57.97 \pm 9.58$ \\
\hline Male:female & $4.5: 1$ \\
\hline Hypertension (\%) & $27(81.8 \%)$ \\
\hline Diabetes mellitus (\%) & $17(51.5 \%)$ \\
\hline Smoking (\%) & $3(0.9 \%)$ \\
\hline Alcoholic (\%) & $12(36.3 \%)$ \\
\hline Chronic kidney disease (\%) & $2(6 \%)$ \\
\hline Angina (\%) & $31(93.9 \%)$ \\
\hline Exertional dyspnea (\%) & $2(6.1 \%)$ \\
\hline
\end{tabular}


after-adenosine FFR values were statistically significant, correlated with $p=0.000(\boldsymbol{- F i g .} \mathbf{1})$.

The mean RFR in our study population was $0.91 \pm 0.07$, FFR basal value of $0.92 \pm 0.06$, and FFR postadenosine 0.87 \pm 0.01 , which were all above the therapeutic index for PCI (-Table 3).

We compared the RFR values with the basal FFR values in all the study populations. When regression analysis was performed for these two variables, both were very well statistically significantly correlated with $p=0.000$ ( - Fig. 2).

We also performed a subgroup analysis in the study population. We compared RFR and FFR values in patients with or without diabetes. When compared in the diabetic study population, RFR with basal FFR $p$-value was 0.000 and RFR with postadenosine FFR $p$-value was 0.001 . In the nondiabetic population, when RFR was compared with basal FFR, the $p$-value was 0.000 ; and the $p$-value for the comparison of RFR with postadenosine FFR was 0.001 . There was no statistically significant difference observed in both the subgroups ( - Fig. $\mathbf{3}$ ).

Table 2 Laboratory parameters of the study population

\begin{tabular}{|l|l|}
\hline Variable & $\begin{array}{l}\text { Mean } \pm \text { standard } \\
\text { deviation }\end{array}$ \\
\hline Hemoglobin $(\mathrm{g} / \mathrm{dL})$ & $13.3 \pm 1.5$ \\
\hline Total leucocyte count $\left(\right.$ cell $\left./ \mathrm{mm}^{3}\right)$ & $6345 \pm 1342$ \\
\hline Creatinine $(\mathrm{mg} / \mathrm{dL})$ & $0.97 \pm 0.32$ \\
\hline Urea $(\mathrm{mg} / \mathrm{dL})$ & $29.58 \pm 9.42$ \\
\hline Hs-CRP $(\mathrm{mg} / \mathrm{dL})$ & $5.845 \pm 1.508$ \\
\hline
\end{tabular}

Abbreviation: Hs-CRP, high-sensitivity C-reactive protein.

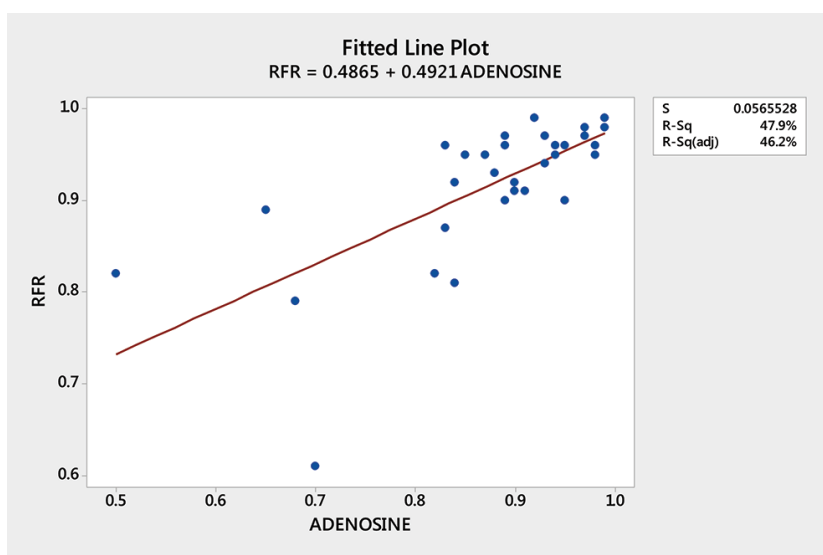

Fig. 1 Comparison of resting full cycle ratio (RFR)with postadenosine fractional flow reserve (FFR) in the study population.

Table 3 Resting full cycle ratio (RFR)and fractional flow reserve (FFR) mean values of the study population

\begin{tabular}{|l|l|l|}
\hline Variable & Mean & $\begin{array}{l}\text { Standard } \\
\text { deviation }\end{array}$ \\
\hline RFR & 0.9188 & 0.0771 \\
\hline FFR basal & 0.9285 & 0.0601 \\
\hline FFR post adenosine & 0.8785 & 0.01084 \\
\hline
\end{tabular}

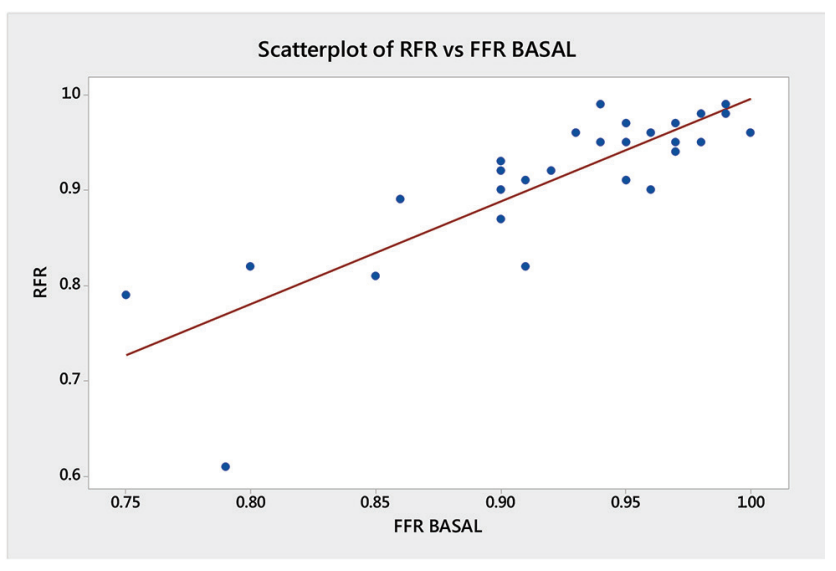

Fig. 2 Comparison of resting full cycle ratio (RFR) with basal fractional flow reserve (FFR)in the study population.

Another subgroup analysis was performed in the female and male population; RFR values were compared with basal and postadenosine FFR. In both the subgroups, no statistically significant difference was observed. In our study population RFR values in both the genders were well correlated with FFR for diagnosing inducible ischemia $(p=0.001)$ ( - Fig. 4).

In our study population, patients who presented with chronic stable angina, after RFR and FFR evaluation, only 4 (12.1\%) patients required percutaneous coronary intervention. In the other 29 (87.9\%) patients, we deferred stenting. However, in one patient who presented with chronic stable angina with normal LV function with RFR value of 0.81 , which is significant but based on FFR values (basal 0.85 , postadenosine 0.84 ), which were not significant, we deferred stenting. When RFR value was more than 0.89 stenting was deferred in all the cases except one where FFR postadenosine value was 0.65 (significant) and the patient was symptomatic; thus, stenting was done in this patient. Apart from these two cases, in all other cases, FFR and RFR values were well correlated, and we used both the values in clinical decision making.

\section{Discussion}

Out of FFR, RFR, IFR available for hemodynamic assessment of coronary lesion severity, FFR is considered better. A large number of studies have been done on RFR and IFR for assessment on coronary lesion severity. Theoretically, out of all, RFR is superior as it does not depend on few segments of cardiac cycle, is independent of ECG, and does not need a coronary hyperemic agent. ${ }^{3}$ RFR has been studied in recent years over 2,000 patients approximately in more than 3,500 coronary lesions. ${ }^{4}$

These studies considered a threshold of 0.89 for RFR. That means if RFR value $<0.89$, the lesion severity is considered significant and intervention in these patients may be beneficial in long-term cardiovascular outcome.

Fractional flow reserve is the ratio of pressure in coronaries distal to the lesion and proximal to the lesion. Its normal value is 1 .To consider the lesion as hemodynamically significant its value should be less than 0.75 . FFR value more than 0.85 is considered as a hemodynamically nonsignificant 


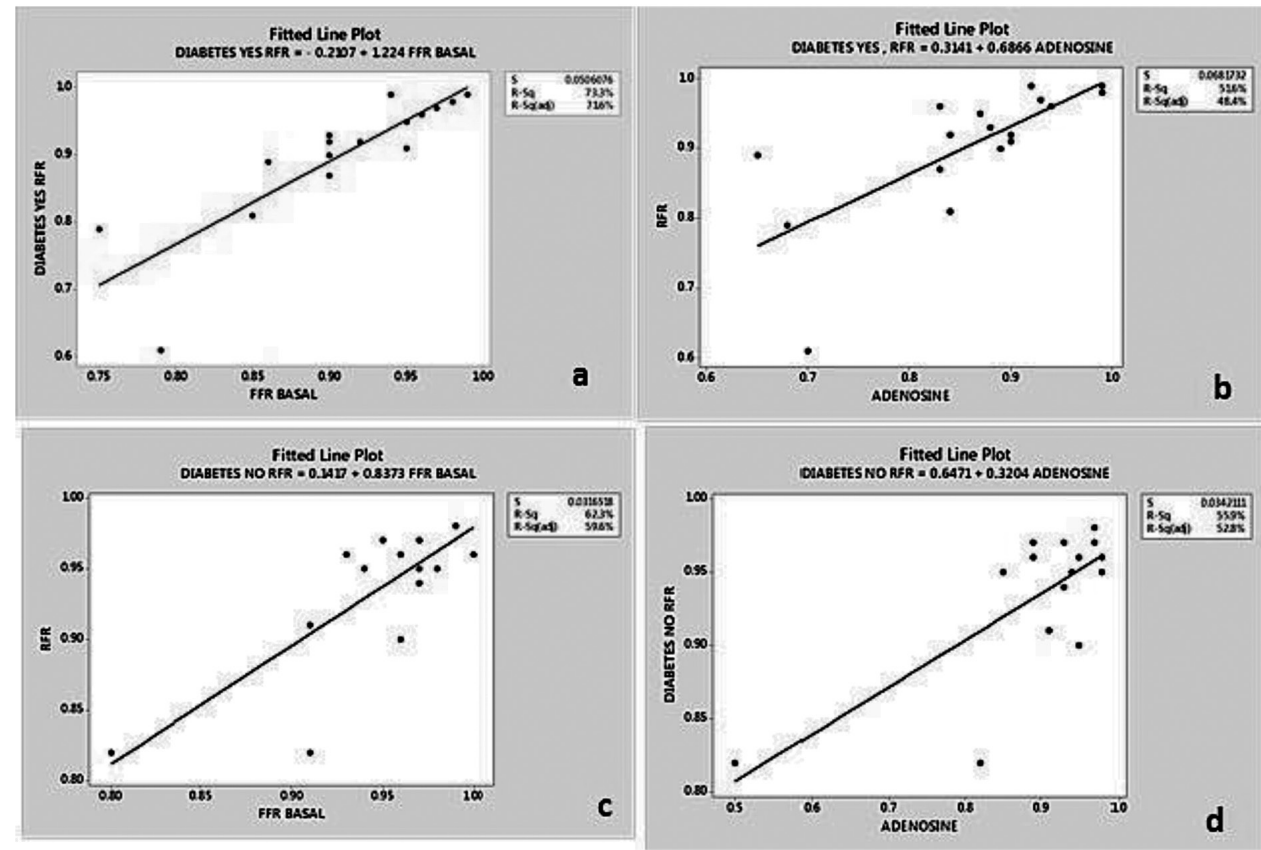

Fig. 3 Comparison of resting full cycle ratio (RFR) with basal fractional flow reserve (FFR)(a), postadenosine FFR (b) in diabetics; RFR with basal FFR (c), postadenosine FFR (d) in nondiabetics.

lesion. When the value is between 0.75 and 0.80 , the decision for intervention should depend on the case basis and clinical judgment.

Studies conducted on FFR concluded that if its value is $>0.75$ stenting is not going to alter the cardiovascular outcomes, but if it is $<0.75$, intervention not done the cardiovascular outcome is poor. So from this observation FFR is used as a tool for decision making for intervention. ${ }^{5}$

IFR is another tool used for hemodynamic assessment of coronary lesion severity. It is a diastolic resting index. It depends on microvascular resistance and myocardial perfusion also. ${ }^{6}$

The existing studies which compared IFR with FFR suggested that there is a good correlation between them statistically for lesion severity hemodynamic assessment. Few studies exist on RFR and IFR, which concluded both were equally effective in diagnosis of severity of coronary lesion. ${ }^{7}$

A substudy conducted in over 435 patients suggested that RFR and IFR have same diagnostic accuracy in predicting cardiovascular outcomes. ${ }^{8}$

Recent study done in acute myocardial infarction patients comparing RFR with FFR observed that correlation between RFR and FFR was 0.84 , overall agreement was $82 \%{ }^{9}$

Studies comparing FFR and RFR are still lacking, so we conducted a study on 33 patients who were undergoing physiological assessment FFR to identify the significance of lesion; RFR values were also taken. Study population mean age was $57.97 \pm 9.58$ years, and it was a predominantly male population.

Our study has compared RFR with FFR for both basal and after-adenosine values. RFR and basal and after-adenosine
FFR values were statistically significantly correlated with each other. When RFR value was of more than 0.89 , stenting was deferred in all the cases except in one case where FFR postadenosine value was 0.65 (significant) and the patient was symptomatic; thus, stenting was done in that patient. In one patient who presented with chronic stable angina with normal LV function whose RFR value of 0.81 , which is significant, but based on FFR values (basal 0.85 , postadenosine 0.84 ), which were not significant, we deferred stenting. Apart from these two cases, in all other cases FFR and RFR values were well correlated and can be used for physiological assessment of inducible ischemia in angiographically proven borderline coronary lesions.

Moreover, subgroup analysis of gender and presence of diabetes was performed in the study population. Both RFR and FFR values were significantly correlated, irrespective of gender and diabetes as a risk factor.

\section{Limitation of the Study}

Two major limitations were there. One is the small study population, small sample for subgroup analysis, and another one was that follow-up of these patients were not done to correlate with events, which is the primary objective of differing PCI depending on the FFR values.

\section{Conclusion}

From this study, we conclude that RFR may be used instead of FFR for physiologic assessment of inducible ischemia in borderline coronary artery lesions as it is independent of gender, risk factor, and cardiac cycle; there is no need to use of a hyperemic agent; and is safe in asthmatic population also. 

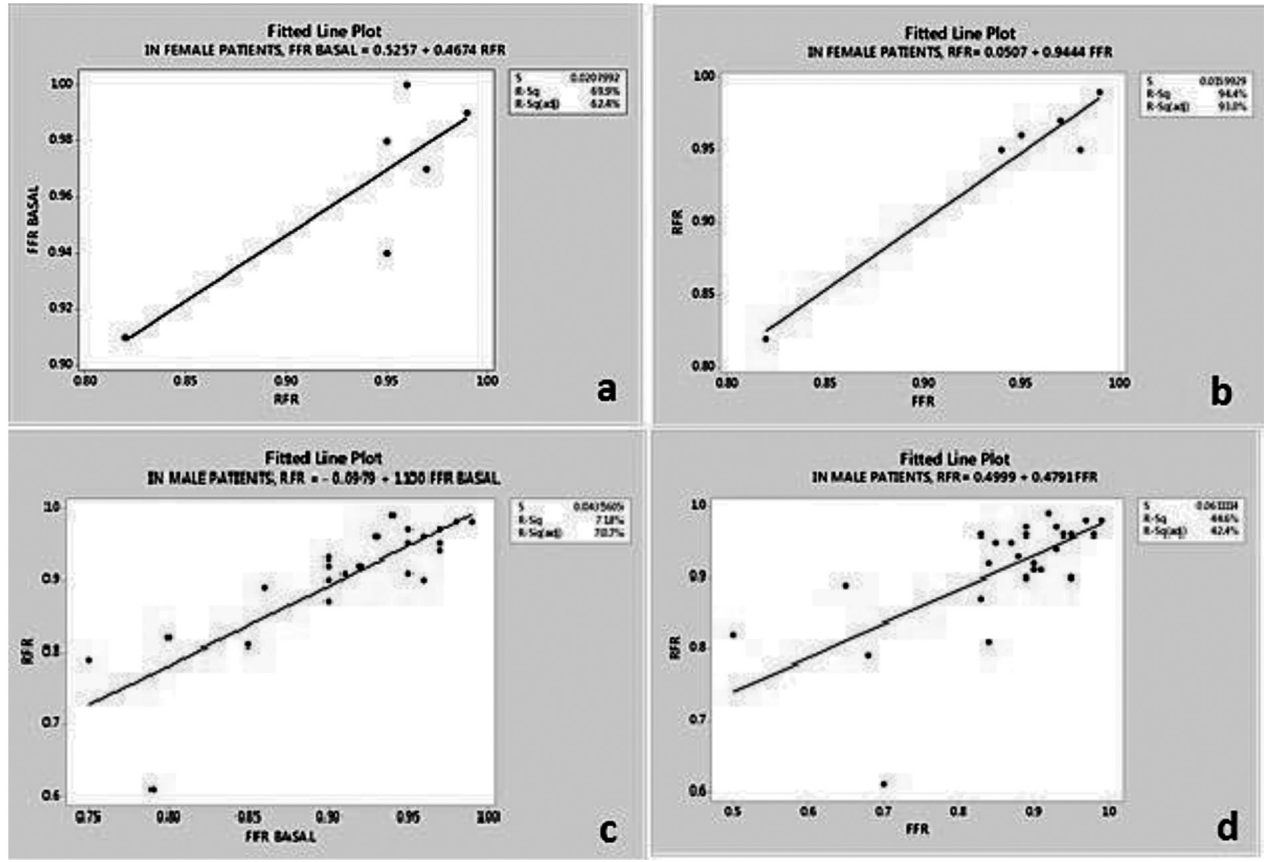

Fig. 4 Comparison of resting full cycle ratio (RFR) with basal fractional flow reserve (FFR) (a), postadenosine FFR (b) in the female; RFR with basal FFR (c), postadenosine FFR (d) in the male population.

\section{Audio File}

Audio file for this article is available at https://doi.org/ 10.1055/s-0040-1709257.

\section{Conflict of Interest}

None declared.

\section{References}

1 Hennigan B, Oldroyd KG, Berry C, et al. Discordance between resting and hyperemic indices of coronary stenosis severity: the VERIFY 2 study. Circ Cardiovasc Interv 2016;9(11):e004016

2 Ahn JM, Park DW, Shin ES, et al; IRIS-FFR Investigators†. Fractional flow reserve and cardiac events in coronary artery disease: data from a prospective IRIS-FFR registry. Circulation 2017;135(23):2241-2251

3 Svanerud J, Ahn JM, Jeremias A, et al. Validation of a novel non-hyperaemic index of coronary artery stenosis severity: the resting full-cycle ratio (VALIDATE RFR) study. EuroIntervention 2018;14(7):806-814

4 Svanerud J, Ahn JM, Jeremias A, et al. Validation of a novel non-hyperaemic index of coronary artery stenosis severity:
The resting full-cycle ratio study. Euro Interven 2018;14(7): 806-814

5 Pijls NH, van Schaardenburgh P, Manoharan G, et al. Percutaneous coronary intervention of functionally nonsignificant stenosis: 5-year follow-up of the DEFER Study. J Am Coll Cardiol 2007;49(21):2105-2111

6 Sen S, Escaned J, Malik IS, et al. Development and validation of a new adenosine-independent index of stenosis severity from coronary wave-intensity analysis: results of the ADVISE (ADenosine Vasodilator Independent Stenosis Evaluation) study. J Am Coll Cardiol 2012;59(15):1392-1402

7 Berry C, van 't Veer M, Witt N, et al. VERIFY (VERification of Instantaneous Wave-Free Ratio and Fractional Flow Reserve for the Assessment of Coronary Artery Stenosis Severity in EverydaY Practice): a multicenter study in consecutive patients. J Am Coll Cardiol 2013;61(13):1421-1427

8 Lee J-M, Koo BK, Shin ES, et al. Clinical implications of threevessel fractional flow reserve measurement in patients with coronary artery disease. Eur Heart J 2018;39(11):945-951

9 Tamargo Delpon MA, Gutierrez E, Diez-Delhoyo F, Gonzalez Saldivar H, Rivera AR, Casado A, et al. Diagnostic accuracy of resting full-cycle ratio in the non-culprit artery of acute patientsnon-culprit artery of acute patients. Eur Heart 2019;40 (Suppl 1):ehz746.0565 